\title{
Mathematical models of the electric arc of variable geometrical parameters based on static voltage-current characteristics
}

\author{
Antoni Sawicki ${ }^{*}$ \\ Czestochowa University of Technology, Faculty of Electrical Engineering, Al. Armii Krajowej 17, \\ 42-200 Czestochowa, Poland
}

\begin{abstract}
The paper presents the Schellhase and Pentegov models utilizing static voltage-current characteristics for representing processes occurring in the electric arc. In the approximations of these characteristics variation in geometrical parameters, i.e. arc length and cross-section is taken into account. A modification of the Voronin-type models is also proposed, introducing a static voltage-current characteristic. In this way, it is possible to take into account distortions of the arc geometrical parameters of the static and dynamic character.
\end{abstract}

\section{Introduction}

The electrical, thermal and gas-dynamic processes occurring in electric arcs are extremely complex therefore several mathematical models have been proposed to account for them. The models are characterized by varying degrees of complexity and accuracy in representing real processes, by different possibilities of identifying characteristics and parameters, and also by different flaws and limitations of applicability, and hence by different universality, usefulness and educational value. In the engineering practice, a few mathematical models have gained reputation for providing relatively accurate and effortless representation of the processes occurring in the working space of electrotechnological devices or in circuits supplying free arcs, whether stabilized or intensely distorted. In the case of some devices and power switches it is relatively easy to obtain static characteristics of an arc supplied from a DC source. But the applicability of such characteristics in classic models used for simulating processes in AC-supplied circuits was highly limited. New mathematical models were subsequently developed, such as the Schellhase and Pentegov models, which meet the basic physical requirements accurately or at least approximately. Since in the modeling of arc static characteristics it is not difficult to take into account distortions of various kinds, the models so obtained are relatively simple and universal. The way of taking distortions into account can be static or dynamic. In the majority of electrotechnological devices the intentional distortions of arc geometrical parameters, used as control actions, are typically gradual and that is why they can be considered static with

* Corresponding author: sawickia@el.pcz.czest.pl 
respect to the speed of electrical processes occurring in the circuits. In power switches, on the other hand, the distortions of the geometrical arc parameters should be very fast. This distinction should be taken into consideration in mathematical models [1].

\section{Dynamic models of the electric arc based on static voltage- current characteristics}

A modified Mayr model of the arc column [2,3] can be represented in a general conductance form

$$
\frac{1}{g} \frac{d g}{d t}=\frac{1}{\theta_{M}}\left[\frac{P_{c o l}(t)}{P_{d i s}(t)}-1\right]
$$

where: $\theta_{M}$ - time of relaxation of the thermal processes; $P_{d i s}$ - power dissipated from the arc column; $P_{c o l}-$ supplied electric power equal to

$$
P_{c o l}(t)=u_{c o l} i=\frac{i^{2}}{g}
$$

In the classic Mayr model it is assumed that $P_{\text {dis }}(t)=P_{M}=$ const. In the range of high current this condition is not met and the Cassie model is typically applied. Since heat dissipation processes respond to external distortions with some time delay, it can be approximately assumed that dissipated power is mostly determined by static characteristics [2] $P_{\text {dis }}(t) \approx P_{\text {stat }}(i(t))$ i.e.

$$
\frac{1}{g} \frac{d g}{d t}=\frac{1}{\theta(i)}\left[\frac{u_{c o l} i}{P_{\text {stat }}(i)}-1\right]
$$

Experimental studies demonstrate that the real damping factor is not a constant value, but it is a function of current $\theta_{M}=\theta(g(i))$. The function of power dissipation is typically approximated by static voltage-current characteristics, in which case

$$
P_{\text {dis }}(t) \approx U_{\text {stat }}(i) \cdot i=\frac{i^{2}}{G_{\text {stat }}(i)}
$$

After substituting (2) and (4) to (3), a modified Mayr equation can be obtained in a conductance form

$$
\frac{1}{g} \frac{d g}{d t}=\frac{1}{\theta_{M}}\left[\frac{G_{\text {stat }}(i)}{g}-1\right]
$$

Now when conductance does not vary over time, the static arc characteristic becomes

$$
\begin{array}{r}
U_{\text {stat }}(i)=\frac{P_{\text {stat }}(i)}{i} \\
G_{\text {stat }}(i)=\frac{i^{2}}{P_{\text {stat }}(i)}=\frac{i^{2}}{U_{\text {stat }}(i) \cdot i}
\end{array}
$$


Then, on the basis of this dependence, the model (5) can be represented in the Schellhase conductance form $[2,4,5]$

$$
\frac{1}{g} \frac{d g}{d t}=\frac{1}{\theta_{S h}}\left[\frac{i}{g \cdot U_{\text {stat }}(i)}-1\right]
$$

Due to the simplifying assumptions specified above, the Schellhase model does not strictly satisfy the power balance equation. At the same time, it is incompatible with other models that satisfy this condition [6] and cannot be considered as their special case.

A generalized arc model that satisfies the power balance equation is the one proposed by Pentegov and subsequently developed jointly with Sidorec [6, 7]. On this approach, instead of a real arc a hypothetical arc is considered, in which resistance is determined on the basis of virtual current $i_{\theta}(t)$, delayed with respect to current $i$, and alternating with a given time constant $\theta$. The square of the virtual current $i$ and the square of the real arc current $i$ are tied by a first-order linear differential equation

$$
\theta \frac{d i_{\theta}^{2}}{d t}+i_{\theta}^{2}=i^{2}
$$

The static voltage-current characteristics of the arc column can be approximated by various functions. Typically, power functions are applied for this purpose. In a general case, arc voltage is

$$
u_{c o l}=R_{s t}\left(i_{\theta}\right) i=\frac{U_{s t a t}\left(i_{\theta}\right)}{i_{\theta}} i
$$

\section{Approximations of the static voltage-current characteristics, taking into account geometrical parameter distortions Equations and mathematics}

The influence of the distortions of geometrical parameters on the static arc characteristics depends on the conditions of energy dissipation:

At first, the case of the free arc will be considered. If current is small, then its variation causes changes in the temperature distribution in the arc column of a constant cross-section area $S(I) \approx$ const. Increase in temperature is accompanied by changes in the plasma parameters, including increase in conductivity, and then $U(I) \propto I^{-1}$. If current is sufficiently high, the plasma temperature reaches a value close to the maximal one and its physical parameters vary only slightly, so current density is nearly constant $(S \propto I)$. This leads to increase in conductance and then $U(S(I)) \approx$ const. Variation in current causes variation in the arc cross-section area, which in turn affects power dissipation from the column lateral surface $P_{d i s} \propto l S^{1 / 2}(I)$.

The second case to be considered is that of a constricted arc. If current is constant, the arc plasma can be constricted $S(t)=$ variab by increasing heat transfer to the surrounding gas or by intensive cooling of the walls [8]. Increase in energy transfer $P_{d i s} \propto l S^{-1 / 2}$ causes increase in arc voltage $U(S(I)) \propto l S^{-1 / 2}$.

Arc length $l$ has influence on arc voltage $\left(U_{\text {stat }} \propto l\right)$. For the free arc, it can be represented with a modified Nottingham formula [9] 


$$
U_{\text {stat }}(I, S(I), l)=\frac{C_{l}+D_{l} l}{I^{m}}+E_{l} l+F_{l} I^{n}
$$

where: $C_{l}, D_{l}, E_{l}, F_{l}, m, n$ - parameters of static characteristics approximation. The first term of this formula is significant in the low current range whereas the last one in the high current range, especially in cases with high pressure and/or pointed electrode.

The impact of the variation in the cross-section area on voltage in a constricted arc $\left(U_{\text {stat }}\right.$ $\left.\propto d^{-1} \propto S^{-1 / 2}\right)$ can be expressed as

$$
U_{\text {stat }}(I, S)=\frac{C_{S}+D_{S} S^{-1 / 2}}{I^{m}}+E_{S} S^{-1 / 2}+F I^{n}
$$

where: $C_{S}, D_{S}, E_{S}, F_{S}, m, n$ - parameters of static characteristics approximation; $d=\sqrt{4 S / \pi} \propto S^{1 / 2}$ - arc column diameter. The resultant arc voltage includes the sum of voltage drops near the electrodes $U_{A K}$.

Since the above-stated formulas do not define an approximate voltage of restriking an $\mathrm{AC}$ arc, sometimes other, more accurate approximations are used $[4,9,10]$, for instance the following

$$
U_{\text {col }}(I)=U_{p} \cdot\left(\frac{I}{I_{p}}\right) \cdot \exp \left(-\left(\frac{I}{I_{p}}\right)+1\right)
$$

where: $I_{p}, U_{p}$ - approximate coordinates of the restriking point. By expanding the formula, it is possible to take into account the constant voltage component $U_{0}$ and the rising component $R I^{k_{0}}$, leading to a modified voltage-current characteristic

$$
\begin{aligned}
& U_{\text {col }}(I, l, S)=\left(U_{0}+R_{0} I^{k_{0}}\right) \cdot \zeta(I)+ \\
& +\left[U_{p}-\left(U_{0}+R_{0} I^{k_{0}}\right) \cdot \zeta(I)\right] \times\left(\frac{I}{I_{p}}\right)^{k_{1}} \cdot \exp \left(-\left(\frac{I}{I_{p}}\right)^{k_{1}}+1\right)
\end{aligned}
$$

where: $\zeta(I)=1-\exp \left(-I / I_{p}\right)$ - weight function; $k_{0} \approx 0.8-1.2,0<k_{1}<1-$ approximation coefficients.

Variation in the column size affects particular approximation parameters. For changes in the arc column length, the following formulas apply:

- strike voltage

$$
U_{p}(l)=E_{p 0} \cdot l^{k_{2}}
$$

- voltage in the high current range

$$
U_{0}(l)=U_{0 m}+u_{0} \cdot l
$$

- resistance in the high current range

$$
R_{0}(l)=R_{0 m}+r_{0} \cdot l
$$


where: $U_{0 m}-$ minimal voltage, $R_{0 m}-$ minimal resistance; $k_{2}, E_{p 0}-$ approximation coefficients dependent on the shape, temperature and material of the electrodes and on the chemical composition of the gas $\left(0<k_{2}<1\right)$. For short arcs in electrotechnological devices, the dependence can be treated as linear $U_{p}(l)=E_{p 0} \cdot l$.

The opposite influence on the parameters of the static characteristic is exerted by distortions of the cross-section in a constricted arc [8]. The relevant formulas are - strike voltage

$$
U_{p}(S)=\frac{E_{p 0}}{S^{k_{2} / 2}}
$$

- voltage in the high current range

$$
U_{0}(S)=U_{0 m}+\frac{u_{0}}{S^{1 / 2}}
$$

- resistance in the high current range

$$
R_{0}(S)=R_{0 m}+\frac{r_{0}}{S^{1 / 2}}
$$

In some mathematical models, such as the modified Mayr model [11], or the hybrid model [12], the minimal value of conductance is defined. In this way it is not necessary to stipulate a precondition on the static characteristics.

A modified Schellhase model for simulating processes in circuits with distortions of arc length and cross-section area can take the following general form

$$
\frac{1}{g} \frac{d g}{d t}=\frac{1}{\theta(g)}\left[\frac{i}{g \cdot U_{\text {stat }}(i, l, S)}-1\right]
$$

In a similar case, arc voltage in the Pentegov model is

$$
u_{c o l}\left(i, i_{\theta}, l, S\right)=\frac{U_{\text {stat }}\left(i_{\theta}, l, S\right)}{i_{\theta}} i
$$

The value of the sum of voltage drops near the electrodes does not practically depend on current. To some extent, the voltage drops depend on the properties of electrode materials, on the shape and temperature of electrodes and on the kind of gas. Typically of the magnitude of about 10-20 Volts, they are commensurate with voltages on short welding arcs so they should be taken into account in analyses of devices employing this kind of an arc. In analyses of long arcs in electrotechnological devices or high-voltage power devices, voltage drops are neglected and approximations include the resultant voltage only. 


\section{Dynamic models of the electric arc of variable geometrical parameters based on distorted static voltage-current characteristics}

Assuming that the dissipation of thermal power is proportional to the lateral area of the arc column and taking as a departure point the power balance equation of an arc with distorted geometrical parameters $S(t)$ and $l(t)$, it is possible to obtain the Voronin model [1]

$$
\frac{1}{g} \frac{d g}{d t}=\frac{1}{\theta_{S}(S)}\left(\frac{u_{c o l} i}{P_{S}(l, S)}-1\right)-\frac{1}{l} \frac{d l}{d t}\left(1+\ln \frac{g l}{\sigma_{0} S}\right)+\frac{1}{S} \frac{d S}{d t}\left(1-\ln \frac{g l}{\sigma_{0} S}\right)
$$

where:

- damping function

$$
\theta_{S}(S)=\frac{q_{0}}{p_{S}} \sqrt{\frac{S}{4 \pi}}
$$

- dissipated power

$$
P_{S}(S)=p_{S} l \sqrt{4 \pi S}
$$

where: $p_{S}-$ density of power dissipated from the arc lateral surface; $\sigma_{0}$ and $q_{0}-$ approximation coefficients of the plasma conductance function. The three parameters are obtained experimentally and treated as constant.

Assuming that the thermal power dissipation is proportional to the arc column volume and starting off with the power balance equation of an arc with distorted geometrical parameters $S(t)$ and $l(t)$, it is possible to arrive at the model [13]

$$
\frac{1}{g} \frac{d g}{d t}=\frac{1}{\theta_{V}}\left(\frac{u_{c o l} i}{P_{V}(l, S)}-1\right)-\frac{1}{l} \frac{d l}{d t}\left(1+\ln \frac{g l}{\sigma_{0} S}\right)+\frac{1}{S} \frac{d S}{d t}\left(1-\ln \frac{g l}{\sigma_{0} S}\right)
$$

where the following notation holds:

$$
\begin{aligned}
& \theta_{V}=\frac{q_{0}}{p_{V}} \\
& P_{V}(l, S)=p_{V} l S
\end{aligned}
$$

where: $p_{V}-$ volumetric density of power dissipated from the arc column.

Tying the two previous conditions of thermal power dissipation, which is proportional to the lateral area, and volume of the arc column and again starting off with the power balance equation of an arc with distorted geometrical parameters $S(t)$ and $l(t)$, it is possible to obtain the model

$$
\frac{1}{g} \frac{d g}{d t}=\frac{1}{\theta_{S V}(S)}\left(\frac{u_{c o l} i}{P_{S V}(l, S)}-1\right)-\frac{1}{l} \frac{d l}{d t}\left(1+\ln \frac{g l}{\sigma_{0} S}\right)+\frac{1}{S} \frac{d S}{d t}\left(1-\ln \frac{g l}{\sigma_{0} S}\right)
$$

with the following notation:

$$
\theta_{S V}(S)=\frac{q_{0}}{p_{V}+p_{S} \sqrt{\frac{4 \pi}{S}}}
$$




$$
P_{S V}(l, S)=l S\left(p_{V}+p_{S} \sqrt{\frac{4 \pi}{S}}\right)
$$

In simplified arc models the damping function is often assumed to be constant $\theta(S)=$ const [14-16]. If the static voltage-current characteristics is introduced into the formula for the dissipated power, in an analogous way as into the Mayr model, then models (23), (26) or (29) can be transformed into an equation of the following general form

$$
\frac{1}{g} \frac{d g}{d t}=\frac{1}{\theta}\left(\frac{i}{g U_{\text {stat }}(i, l, S)}-1\right)-\frac{1}{l} \frac{d l}{d t}\left(1+\ln \frac{g l}{\sigma_{0} S}\right)+\frac{1}{S} \frac{d S}{d t}\left(1-\ln \frac{g l}{\sigma_{0} S}\right)
$$

Due to the above-mentioned simplifying assumptions, model (32) does not strictly satisfy the power balance equation. It is not compatible with models (23), (26) and (29), which do satisfy this condition, and cannot be treated as their special case. If it is assumed that the variation in the geometrical parameters occurs slowly $(d l / d t \approx 0, d S / d t \approx 0)$, then model (32) can be transformed into the Schellhase model (21).

\section{Simulations of processes occurring in arc circuits using models of arcs with distorted parameters}

For slow distortions of arc column parameters, the Schellhase or Pentegov models are typically applied. In some devices however, the changes in the working conditions occur very fast, which may lead to deteriorating the discharge stability and to undesirable changes in technological effects. This indicates the conditions with rapid changes have to be taken into account in simulation tests $[1,17]$.

The initial assumptions of the Voronin model [1] stipulate that the influence of current on the Gaussian temperature distribution and on other plasma parameters in the arc crosssection are taken into account, which makes the static characteristics hyperbolic. Such conditions correspond best with a low-current arc and can be accounted for by means of the Mayr model, together with some simplifying assumptions. The modified variant of the Voronin model (32) is free from such limitations and its static characteristic can take any shape.

The first part of the simulation experiment involved obtaining graphs representing static voltage-current characteristics corresponding to modified Nottingham formulas for an extended arc and for a constricted arc. The results are presented in Fig.1. Fig. 2 presents families of static voltage-current characteristics corresponding to formulas including a defined value of restrike voltage for an extended arc and for a constricted arc. These characteristics were used for modeling an arc in a circuit with sinusoidal current of the amplitude $200 \mathrm{~A}$ and frequency $50 \mathrm{~Hz}$. The respective dynamic characteristics are presented in Figs 3 and 4. The simulations took into account the voltage drop near the electrodes equal to $U_{A K}=18 \mathrm{~V}$. Due to a possible form and values of parameters of the approximation functions, the general variant of the calculations should take into consideration the static characteristic $U_{\text {stat }}(|i|, l, S) \cdot \operatorname{sgn} i$. 
a)

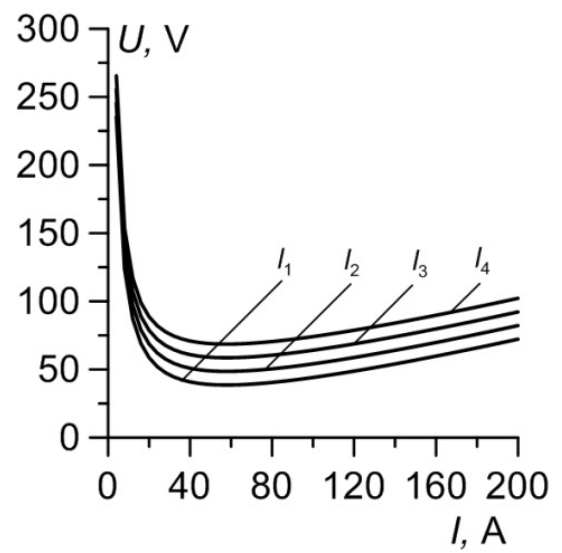

b)

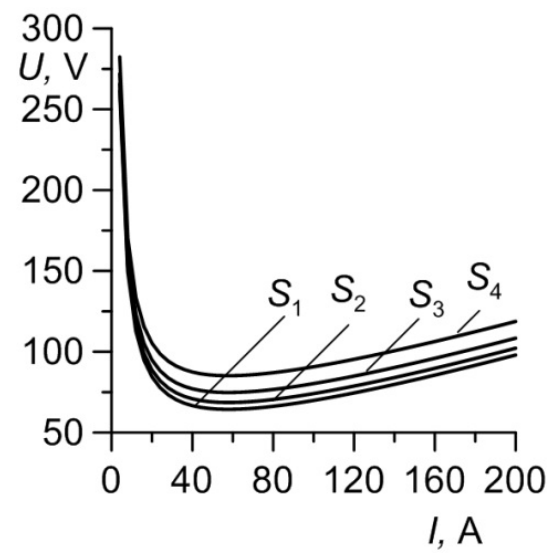

Fig.1. Family of static voltage-current characteristics corresponding to modified Nottingham formulas, with undefined restrike voltage: a) formula (11) for an extended $\operatorname{arc}\left(C_{l}=900 \mathrm{VA}^{m}, D_{l}=10\right.$ $\mathrm{VA}^{\mathrm{m}} / \mathrm{m}, m=1, E_{l}=2 \cdot 10^{3} \mathrm{~V} / \mathrm{m}, F_{l}=0,1 \mathrm{~V} / \mathrm{A}^{\mathrm{n}}, n=1,2 ; l_{1}=5 \cdot 10^{-3} \mathrm{~m}, l_{2}=10 \cdot 10^{-3} \mathrm{~m}, l_{3}=15 \cdot 10^{-3} \mathrm{~m}, l_{4}$ $\left.=20 \cdot 10^{-3} \mathrm{~m}\right)$; b) formula (12) for a constricted $\operatorname{arc}\left(C_{S}=900 \mathrm{VA}^{\mathrm{m}}, D_{S}=0,02 \mathrm{VAm}^{0,5}, m=1, E_{S}=0,8\right.$ $\left.\mathrm{Vm}^{0,5}, F_{S}=0,1 \mathrm{~V} / \mathrm{A}^{\mathrm{n}}, n=1,2 ; S_{1}=5 \cdot 10^{-4} \mathrm{~m}^{2}, S_{2}=4 \cdot 10^{-4} \mathrm{~m}^{2}, S_{3}=3 \cdot 10^{-4} \mathrm{~m}^{2}, S_{4}=2 \cdot 10^{-4} \mathrm{~m}^{2}\right)$.

a)

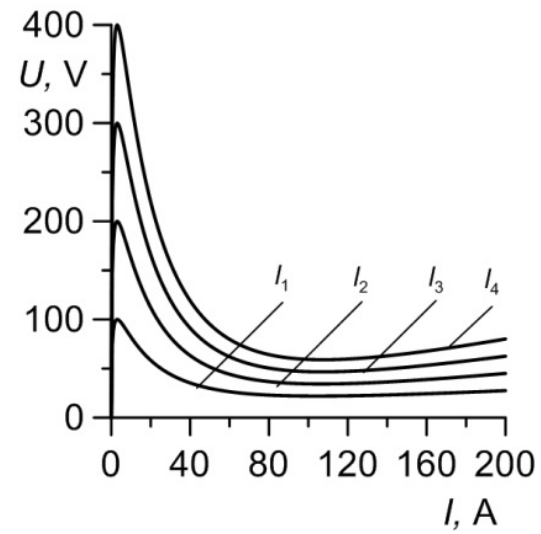

b)

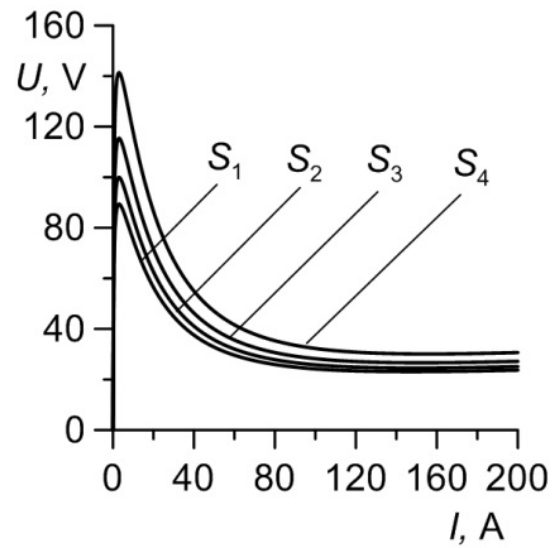

Fig.2. Family of static voltage-current characteristics corresponding to formulas with defined restrike voltage: a) formulas (14)-(17) for an extended $\operatorname{arc}\left(I_{p}=3 \mathrm{~A}, E_{p 0}=20 \cdot 10^{3} \mathrm{~V} / \mathrm{m}^{\mathrm{k} 2}, k_{0}=1,1, k_{1}=0,5, k_{2}\right.$ $=1, U_{0 \mathrm{~m}}=10 \mathrm{~V}, u_{0}=5 \mathrm{~V} / \mathrm{m}, R_{0 \mathrm{~m}}=1 \cdot 10^{-4} \Omega, r_{0}=10 \Omega / \mathrm{m} ; l_{1}=5 \cdot 10^{-3} \mathrm{~m}, l_{2}=10 \cdot 10^{-3} \mathrm{~m}, l_{3}=15 \cdot 10^{-3} \mathrm{~m}$, $\left.l_{4}=20 \cdot 10^{-3} \mathrm{~m}\right)$; b) formulas (14), (18)-(20) for a constricted $\operatorname{arc}\left(I_{p}=3 \mathrm{~A}, E_{p 0}=2 \mathrm{Vm}^{\mathrm{k} 2 / 2}, k_{0}=1,2, k_{1}\right.$ $=0,5, k_{2}=1, U_{0 \mathrm{~m}}=10 \mathrm{~V}, u_{0}=0,2 \mathrm{Vm}^{1 / 2}, R_{0 m}=3 \cdot 10^{-3} \Omega, r_{0}=1 \cdot 10^{-4} \Omega \mathrm{m}^{1 / 2} ; S_{1}=5 \cdot 10^{-4} \mathrm{~m}^{2}, S_{2}=4 \cdot 10^{-4}$ $\left.\mathrm{m}^{2}, S_{3}=3 \cdot 10^{-4} \mathrm{~m}^{2}, S_{4}=2 \cdot 10^{-4} \mathrm{~m}^{2}\right)$. 
a)

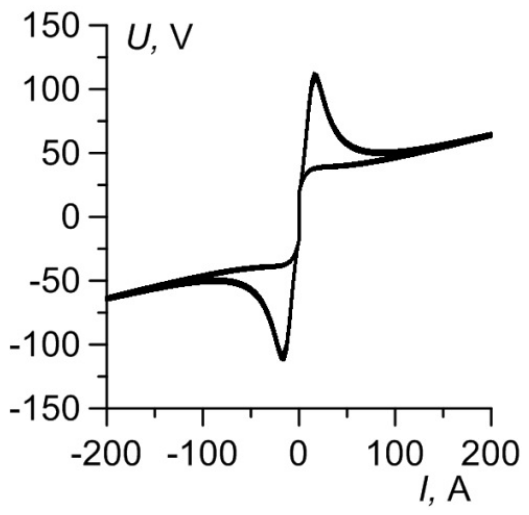

b)

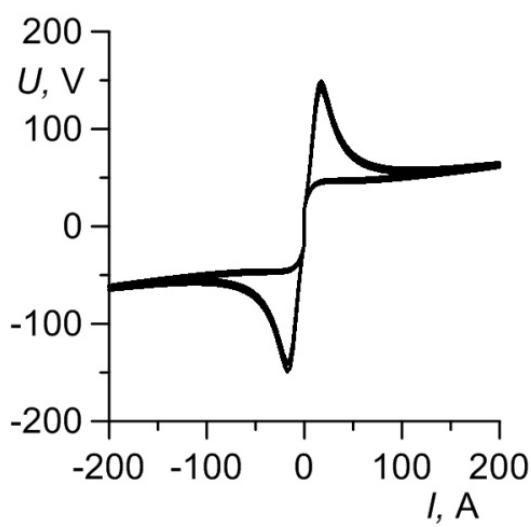

Fig.3. Dynamic voltage-current characteristics corresponding to the modified Voronin-Schellhase formula (8) obtained on the basis of an approximated static characteristic as in a) formula (11) for an extended $\operatorname{arc}\left(C_{l}=800 \mathrm{VA}^{\mathrm{m}}, D_{l}=0,5 \mathrm{VA}^{\mathrm{m}} / \mathrm{m}, m_{l}=1, E_{l}=50 \mathrm{~V} / \mathrm{m}, F_{l}=0,2 \mathrm{~V} / \mathrm{A}^{\mathrm{n}}, n_{l}=1, \theta=2 \cdot 10^{-4} \mathrm{~s}\right.$, $S=5 \cdot 10^{-4} \mathrm{~m}^{2}, l=3 \cdot 10^{-3}+5 \cdot 10^{-2} t$, after $0,5 \mathrm{~s}$ step o $\Delta l=+1 \cdot 10^{-2} \mathrm{~m}$ ); b) formula (12) for a constricted arc $\left(C_{s}=1000 \mathrm{VA}^{\mathrm{m}}, D_{s}=0,5 \mathrm{VA}^{\mathrm{m}} \mathrm{m}^{0,5}, m_{s}=1, E_{s}=0,2 \mathrm{Vm}^{0,5}, F_{s}=0,15 \mathrm{~V} / \mathrm{A}^{\mathrm{n}}, n_{s}=1, \theta=2 \cdot 10^{-4} \mathrm{~s}, l=\right.$ $6 \cdot 10^{-3} \mathrm{~m}, S=8 \cdot 10^{-4} \mathrm{~m}^{2}$ after $0,5 \mathrm{~s}$ step $\Delta S=-5 \cdot 10^{-4} \mathrm{~m}^{2}$ ).

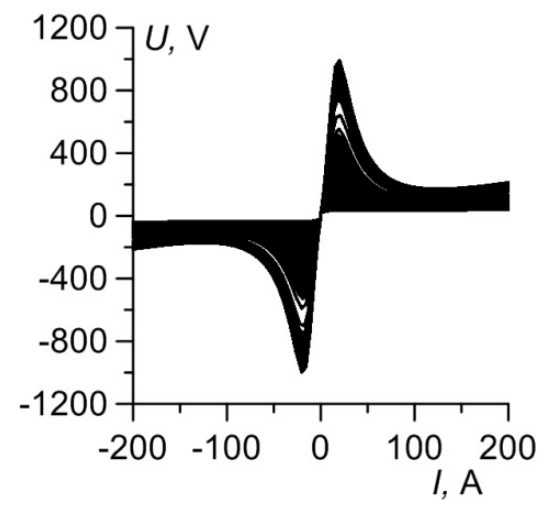

Fig.4. Dynamic voltage-current characteristics corresponding to the modified Voronin-Schellhase formula (8) obtained on the basis of the approximation of the static characteristic given in (14)-(17) for an extended $\operatorname{arc}\left(I_{p}=3 \mathrm{~A}, E_{p 0}=20 \cdot 10^{3} \mathrm{~V} / \mathrm{m}^{k 2}, k_{0}=1,2, k_{1}=0,5, k_{2}=1, U_{0 \mathrm{~m}}=10 \mathrm{~V}, u_{0}=5 \mathrm{~V} / \mathrm{m}\right.$, $R_{0 \mathrm{~m}}=1 \cdot 10^{-4} \Omega, r_{0}=10 \Omega / \mathrm{m}, \sigma_{0}=150 \cdot 10^{3} \mathrm{~S} / \mathrm{m}, \theta=2 \cdot 10^{-4} \mathrm{~s}, S=5 \cdot 10^{-4} \mathrm{~m}^{2}, l=3 \cdot 10^{-3}+5 \cdot 10^{-2} t$, after $0,5 \mathrm{~s}$ step $\left.\Delta l=+1 \cdot 10^{-2} \mathrm{~m}\right)$.

\section{Conclusions:}

1. The Schellhase model of the electric arc offers a possibility of taking into account the static form of distortions in the arc column geometrical parameters.

2. Due to its simplifying assumptions, the classic Voronin model of the electric arc [2, 3, 5] has a limited potential for representing the characteristics on an arc with distorted geometrical parameters.

3. The Voronin model is suitable for introducing various formulas for dissipated power, at the same time offering the possibility of representing the impact of dynamic 
distortions of the geometrical parameters of the arc column on its electric characteristics.

4. When the simplifying assumptions are adapted in the Voronin type models, introducing static arc characteristics deteriorates the adequacy of their physical interpretation, but at the same time significantly improves the possibility of approximating dynamic arc characteristics.

5. The simulations carried out on the modified variants of the Voronin model indicate that they are applicable for representing dynamic characteristics of arcs with distorted geometrical parameters.

\section{References}

1. A.A. Voronin, Povyšenie èffektivnostikontaktno-dugogasitel'nyh sistem sil'notočnyh kommutacionnyh apparatov s udlinâûsejsâ dugoj, Avtoreferat dis. k.t.n. Samara (2009)

2. A. Sawicki, Śląskie Wiadomości Elektryczne 105, 13 (2012)

3. A. Sawicki, Śląskie Wiadomości Elektryczne 105, 9 (2012)

4. L. Marciniak, Archiwum Energetyki 37, 357 (2007)

5. A. Sawicki, Zeszyty Naukowe Politechniki Łódzkiej, Elektryka Nr 124, 202 (2012)

6. I.V. Pentegov, V.N. Sydorets, Avtomatičeskaâ svarka 431, 33 (1989)

7. I.V. Pentegov, Avtomatičeskaâ svarka 279, 8 (1976).

8. A.M. Krouchinin, A. Sawicki, Modelling of the constricted arc in plasma generators, Seria Monografie nr 109, The Publishing Office of Częstochowa University of Technology, Częstochowa (2005)

9. A. Sawicki, Biuletyn Instytutu Spawalnictwa 57, 58 (2013)

10. L. Marciniak, Przegląd Elektrotechniczny 85, 188 (2009)

11. A. Sawicki, Przegląd Elektrotechniczny 93, 58 (2017)

12. A. Sawicki, Przegląd Elektrotechniczny 93, 198 (2017)

13. A. Sawicki, Wiadomości Elektrotechniczne 80, 15 (2012)

14. A. Sawicki, Śląskie Wiadomości Elektryczne 105, 18 (2012)

15.Z. Ciok, Procesy taczeniowe w uktadach elektroenergetycznych, WNT, Warszawa (1976).

16. M. Wciślik, Przegląd Elektrotechniczny 84, 115 (2008)

17. A. Sawicki, Przegląd Elektrotechniczny 88, 196 (2012) 